\title{
Recommendations for reporting tumor budding in colorectal cancer based on the International Tumor Budding Consensus Conference (ITBCC) 2016
}

Alessandro Lugli ${ }^{1,22}$, Richard Kirsch ${ }^{2,22}$, Yoichi Ajioka ${ }^{3}$, Fred Bosman ${ }^{4}$, Gieri Cathomas ${ }^{5}$, Heather Dawson ${ }^{1}$, Hala El Zimaity ${ }^{6}$, Jean-François Fléjou ${ }^{7}$, Tine Plato Hansen ${ }^{8}$, Arndt Hartmann ${ }^{9}$, Sanjay Kakar ${ }^{10}$, Cord Langner ${ }^{11}$, Iris Nagtegaal ${ }^{12}$, Giacomo Puppa ${ }^{13}$, Robert Riddell $^{2}$, Ari Ristimäki ${ }^{14}$, Kieran Sheahan ${ }^{15}$, Thomas Smyrk ${ }^{16}$, Kenichi Sugihara ${ }^{17}$, Benoît Terris $^{18}$, Hideki Ueno ${ }^{19}$, Michael Vieth ${ }^{20}$, Inti Zlobec ${ }^{1}$ and Phil Quirke ${ }^{21}$

${ }^{1}$ Institute of Pathology, University of Bern, Bern, Switzerland; ${ }^{2}$ Pathology and Laboratory Medicine, Mount Sinai Hospital, University of Toronto, Toronto, Ontario, Canada; ${ }^{3}$ Division of Molecular and Diagnostic Pathology, Niigata University Graduate School of Medical and Dental Sciences, Niigata, Japan; ${ }^{4}$ University Institute of Pathology, Lausanne University Medical Center, Lausanne, Switzerland; ${ }^{5}$ Institute of Pathology, Kantonsspital Liestal, Liestal, Switzerland; ${ }^{6}$ Dynacare Laboratory, Brampton, Ontario, Canada; ${ }^{7}$ Pathology Department, Saint-Antoine Hospital, Pierre et Marie Curie University, Paris, France; ${ }^{8}$ Department of Pathology, Copenhagen University Hospital, Herlev, Denmark; ${ }^{9}$ Department of Pathology, University Hospital Erlangen, Erlangen, Germany; ${ }^{10}$ Department of Anatomic Pathology, University of California, San Francisco, CA, USA; ${ }^{11}$ Institute of Pathology, Medical University of Graz, Graz, Austria; ${ }^{12}$ Department of Pathology, Radboud University Medical Centre, Nijmegen, The Netherlands; ${ }^{13}$ Department of Clinical Pathology, Geneva University Hospital, Geneva, Switzerland; ${ }^{14}$ Department of Pathology, University of Helsinki and Helsinki University Hospital, Helsinki, Finland; ${ }^{15}$ Department of Pathology, St Vincent's University Hospital, Dublin, Ireland; ${ }^{16}$ Divisions of Anatomic Pathology and Mayo Clinic, Rochester, MN, USA; ${ }^{77}$ Department of Surgical Oncology, Tokyo Medical and Dental University, Graduate School of Medical and Dental Sciences,

Bunkyo-ku, Tokyo, Japan; ${ }^{18}$ Pathology Department, Hôpital Cochin and Université Paris Descartes Sorbonne Paris Cité, Paris, France; ${ }^{19}$ Department of Surgery, National Defense Medical College, Tokorozawa, Saitama, Japan; ${ }^{20}$ Institute of Pathology, Klinikum Bayreuth, Bayreuth, Germany and ${ }^{21}$ Pathology and Tumour Biology, University of Leeds, St James's University Hospital, Leeds, UK

Tumor budding is a well-established independent prognostic factor in colorectal cancer but a standardized method for its assessment has been lacking. The primary aim of the International Tumor Budding Consensus Conference (ITBCC) was to reach agreement on an international, evidence-based standardized scoring system for tumor budding in colorectal cancer. The ITBCC included nine sessions with presentations, a pre-meeting survey and an e-book covering the key publications on tumor budding in colorectal cancer. The 'Grading of Recommendation Assessment, Development and Evaluation' method was used to determine the strength of recommendations and quality of evidence. The following 10 statements achieved consensus: tumor budding is defined as a single tumor cell or a cell cluster consisting of four tumor cells or less (22/22, 100\%). Tumor budding is an independent predictor of lymph node metastases in pT1 colorectal cancer $(23 / 23,100 \%)$. Tumor budding is an independent predictor of survival in stage II colorectal cancer (23/23, 100\%). Tumor budding should be taken into account along with other clinicopathological features in a multidisciplinary setting $(23 / 23,100 \%)$. Tumor budding is counted on H\&E (19/22, 86\%). Intratumoral budding exists in colorectal cancer and has been shown to be related to lymph node metastasis $(22 / 22,100 \%$ ). Tumor budding is assessed in one hotspot (in a field measuring $\left.0.785 \mathrm{~mm}^{2}\right)$ at the invasive front $(22 / 22,100 \%)$. A three-tier system should be used along with the

Correspondence: Professor A Lugli, MD, Institute of Pathology, University of Bern, Murtenstrasse 31, Bern 3008, Switzerland.

E-mail: alessandro.lugli@pathology.unibe.ch

${ }^{22}$ These authors contributed equally to this work.

Received 23 February 2017; revised 28 March 2017; accepted 29 March 2017; published online 26 May 2017 
budding count in order to facilitate risk stratification in colorectal cancer $(23 / 23,100 \%)$. Tumor budding and tumor grade are not the same $(23 / 23,100 \%)$. Tumor budding should be included in guidelines/protocols for colorectal cancer reporting (23/23, 100\%). Members of the ITBCC were able to reach strong consensus on a single international, evidence-based method for tumor budding assessment and reporting. It is proposed that this method be incorporated into colorectal cancer guidelines/protocols and staging systems.

Modern Pathology (2017) 30, 1299-1311; doi:10.1038/modpathol.2017.46; published online 26 May 2017

The TNM classification system remains the gold standard for stratification of colorectal cancer patients into prognostic subgroups. Nevertheless, heterogeneity in survival within the same tumor stages points to the need for additional prognostic biomarkers. Tumor budding is defined as single cells or clusters of up to four cells at the invasive margin of colorectal cancer ${ }^{1}$ and can be stratified into peritumoral budding (PTB, tumor buds at the tumor front) and intratumoral budding (ITB, tumor buds in the tumor center). ${ }^{2}$ PTB can only be assessed in endoscopic or surgical resection specimens, whereas ITB can be assessed in both colorectal cancer biopsies and resection specimens. Both ITB and PTB are morphologic manifestations of epithelialmesenchymal transition (EMT). A ZEB1, SNAIL1, TWIST1-positive microenvironment is conductive to the tumor budding phenotype. ${ }^{3}$ In addition, tumor buds show loss of the adhesion molecule E-cadherin and express markers of an activated wnt signaling pathway such as nuclear beta-catenin and APC.,5 Furthermore, tumor buds express matrix metalloproteinases, cyclin D1, VEGF and p16, but do not show increased proliferation as determined by MIB1. ${ }^{4,6-9}$ Also of interest is that tumor buds may express stem cell markers, such as EpCAM and ABGC5. ${ }^{10}$ The presence of ABCG5 in buds was associated with a worse prognosis in node-negative colorectal cancer patients. ${ }^{10}$

Tumor budding is an independent adverse prognostic factor in colorectal cancer. ${ }^{11-15}$ It is also associated with a higher TNM stage, high tumor grade, the presence of lymphovascular invasion and consequently with lymph node and distant metastases. ${ }^{11-15}$ In colorectal cancer, tumor budding can potentially be applied as an additional quantitative prognostic factor to facilitate the management of colorectal cancer patients in three clinical scenarios. First, in endoscopically resected pT1 colorectal cancer, tumor budding is associated with an increased risk of lymph node metastases. Therefore, patients with budding may benefit from surgical resection. ${ }^{16-18}$ Second, in stage II colorectal cancer, the presence of tumor budding is an indicator of shorter disease-free survival compared with stage II colorectal cancer with low-grade budding, or no budding. Therefore, stage II colorectal cancer patients with high-grade tumor budding may be considered for adjuvant therapy. ${ }^{13,14,18,19}$ Third, ITB assessed in pre-operative biopsies could help select patients who may qualify for neo-adjuvant therapy and could potentially predict tumor regression. ${ }^{20-22}$

One of the main reasons that tumor budding is not routinely reported is the lack of a standardized scoring system that is simple and reproducible. ${ }^{11,12}$ The selection of the tumor slide, the location of counting, the applied stain (H\&E vs immunohistochemistry) and the scoring system (cut-off vs continuous scale) are practical points that need to be clarified and then validated in multiple studies. Nevertheless, tumor budding seems to be a robust biomarker, which retains its prognostic value independently of selected scoring systems used in different studies. ${ }^{11-15}$ The primary objective of the International Tumor Budding Consensus Conference (ITBCC), which took place in Bern in April 2016, was to reach agreement on an evidence-based, standardized scoring system for tumor budding to be used in international colorectal cancer guidelines and routine practice.

\section{Materials and methods}

\section{Consensus Process}

The ITBCC was initiated by members of the ITBCC steering committee at the annual meeting of the United States and Canadian Academy of Pathology (USCAP) in March 2015 in Boston. The ITBCC was held in 2729 April 2016 in the Kursaal, Bern, Switzerland, and included participants from 11 countries. The primary objective was to determine whether consensus could be reached on a standardized scoring method for assessing tumor budding. The ITBCC included nine sessions with presentations, a pre-meeting survey and an e-book covering the most important publications on tumor budding in colorectal cancer.

\section{Steering Committee and Participants}

Twenty-five participants (22 gastrointestinal pathologists, 2 surgeons and 1 translational researcher) from the United States, Canada, Japan and Europe with expertise in tumor budding were invited to attend the face-to-face meeting in 28-29 April 2016. Two participants could not attend the meeting, but participated in the pre-meeting survey. The steering committee, composed of six participants (AL, RK, RR, HD, GC and IZ), organized the meeting in Bern and prepared the pre-meeting survey and the e-book in collaboration 
Table 1 Pre-meeting survey

Question
1. In which of the following clinical scenarios in colorectal cance
should tumor budding be reported? Select all that apply
2: Regarding stains used to assess tumor budding, which of the
following best describes your practice? Please select one answer

3: Which criteria do you use in practice to define ('high grade') tumor budding? Please select only one answer

4: Regarding the assessment of tumor budding, select only one answer

5: Please indicate your level of agreement/disagreement with the following statement: "the field size for tumor budding should be based on area (measured in $\mathrm{mm}^{2}$ ) rather than the objective lens used (eg, 20x)'. Please select only one answer

6: Please indicate your level of agreement/disagreement with the following statement: 'if universally accepted criteria were available, tumor budding should be a mandatory reportable data element.' Please select only one answer

7: Regarding tumor budding in pT1 colorectal cancers, which best describes the practice of clinical colleagues at your institution? Please select only one answer

8: Regarding tumor budding in stage II colorectal cancer, which best describes the oncologists at your institution? Please select only one answer
Answer

a) PT1 colorectal cancers (24/24, 100\%)

b) Stage II colorectal cancer resection specimen $(23 / 24,96 \%$ )

c) All colorectal cancer resection specimens (13/24, 54\%)

d) Pre-operative biopsies (13/24, 54\%)

e) All colorectal cancer stages and specimens (8/24, 33\%)

a) Assessed on $\mathrm{H} \& \mathrm{E}$ sections only (10/24, 42\%)

b) Assessed on H\&E with IHC limited to confirming challenging or suspicious cases $(10 / 24,42 \%)$

c) Assessed on IHC only $(4 / 24,17 \%)$

d) Other please specify $(0 / 24,0 \%)$

a) $\geq 10$ buds in a single field (20x objective) on $\mathrm{H} \& \mathrm{E}(10 / 24,42 \%$ )

b) $\geq 5$ buds in a single field (20x objective) on $\mathrm{H} \& \mathrm{E}(3 / 24,13 \%)$

c) ' $10 \mathrm{HPF}$ method' using IHC ( $\geq 10 \mathrm{HPF}, 40 \mathrm{x}$ objective) $(0 / 24,0 \%)$

d) No cut-off used (prefer continuous scale, reporting buds/HPF) (4/24,

$17 \%$ )

e) Other (Wang method, Japanese classification of colon and rectum cancer) $(7 / 24,29 \%)$

a) Limited to the invasive front $(11 / 24,46 \%)$

b) Focused on, but not limited to, the invasive front $(10 / 24,42 \%)$

c) Neither focused on nor limited to the invasive front $(3 / 24,13 \%)$

a) Strongly disagree $(0 / 24,0 \%)$

b) Disagree $(3 / 24,13 \%)$

c) Neutral or uncertain $(4 / 24,17 \%)$

d) Agree (9/24, 38\%)

e) Strongly agree $(8 / 24,33 \%)$

a) Strongly disagree $(0 / 24,0 \%)$

b) Disagree (2/24, $8 \%)$

c) Neutral or uncertain $(3 / 24,13 \%)$

d) Agree $(7 / 24,29 \%)$

e) Strongly agree $(12 / 24,50 \%)$

a) NOT taken into account by clinical colleagues in the management of pT1 colorectal cancers $(5 / 24,21 \%)$

b) Taken into account by SOME clinical colleagues in the management of pT1 colorectal cancers $(9 / 24,28 \%)$

c) Taken into account by MOST clinical colleagues in the management of pT1 colorectal cancers $(6 / 24,25 \%)$

d) Taken into account by ALL clinical colleagues in the management of pT1 colorectal cancers $(4 / 24,17 \%)$

a) Not aware of the prognostic importance $(4 / 24,17 \%)$,

b) Somewhat aware of the prognostic importance $(10 / 24,42 \%)$

c) Highly aware of the prognostic importance but not currently

considered in the decision to offer adjuvant chemotherapy in high-risk stage II colorectal cancer $(7 / 24,29 \%)$

d) Highly aware of the prognostic importance and considered in the decision to offer adjuvant chemotherapy in high-risk stage II colorectal cancer $(3 / 24,13 \%)$ with two chairmen (FB and PQ). All 25 participants were voting members and differences in vote numbers are due to participants who were not able to attend the meeting or could only attend on 1 day.

\section{Pre-Meeting Survey}

The nine-question pre-meeting survey was sent to all the participants before the meeting and results were presented during the sessions (summarized in Table 1).

\section{Sessions}

Based on the results of the pre-meeting survey, the ITBCC was organized in nine sessions, with preliminary statements serving as a starting point for discussions (see below):
- Session 1: definitions of tumor budding.

- Statement 1: tumor budding is defined as a single cell or a cell cluster.

- Statement 2: tumor budding is different from poorly differentiated clusters.

- Session 2: clinical scenarios and tumor budding.

- Statement 1: tumor budding is an independent predictor of lymph node metastases in pT1 colorectal cancers.

- Statement 2: tumor budding is an independent predictor of survival in stage II colorectal cancer.

- Statement 3: tumor budding is an adverse prognostic factor in pre-operative biopsies of colorectal cancer.

- Session 3: H\&E and immunohistochemistry for the tumor budding score. 
- Statement 1: tumor budding is assessed on H\&E provided there are no features that limit its assessment (eg, peritumoral inflammation, glandular fragmentation).

- Statement 2: immunohistochemistry, as optimal visualization tool, is applied in cases where $\mathrm{H} \& \mathrm{E}$ assessment is limited.

- Statement 3: on immunohistochemical stained slides, digital software provides an objective budding count.

- Session 4: intratumoral and peritumoral budding.

- Statement 1: ITB is applied on pre-operative biopsies.

- Statement 2: the prognostic impact of tumor budding is independent of its location (ie, ITB vs PTB).

- Statement 3: all tumor buds (ie, ITB+PTB) are counted in pT1 colorectal cancers and stage II colorectal cancers).

- Session 5: field number and size for the tumor budding score.

- Statement 1: tumor budding assessment in preoperative biopsies and pT1 colorectal cancers is performed using the 'hotspot' approach.

- Statement 2: in surgical resection specimens, the 'hotspot' and $10 \mathrm{HPF}$ methods are similar in terms of prognostic information (despite superior reproducibility of the $10 \mathrm{HPF}$ method) and are both used.

- Statement 3: the field size definition is independent of the microscope type used.

- Session 6: cut-offs and continuous scale for the tumor budding score.

- Statement 1: a cut-off for high-grade tumor budding is used in order to facilitate meaningful risk stratification in colorectal cancer.

- Statement 2: upon specific request by the responsible clinician a continuous scale for tumor budding score (which allows more precise risk assessment) is provided.

- Statement 3: the chosen method is sufficiently reproducible.

- Session 7: reporting tumor budding.

- Statement 1: tumor budding should be a standard element in guidelines/protocols for colorectal cancer reporting.

- Statement 2: tumor budding should included in the next TNM classification as an additional prognostic factor equal to $\mathrm{L}, \mathrm{V}$ or Pn stage.

- Statement 3: tumor budding should not be taken into account in the assessment of tumor grade.

- Session 8 and 9: proceedings, conclusions and further studies.

\section{Sources}

A systematic literature search in PubMed for tumor budding in colorectal cancer was performed. An e-book including 38 key publications relevant to specific sessions was circulated to all participants ahead of the meeting: session 1: definitions of tumor budding; ${ }^{14,18,23-30}$ session 2: clinical scenarios and tumor budding, ${ }^{2,17-22,31-40}$ session 3: H\&E and immunohistochemistry for the tumor budding score; ${ }^{11-13,41-46}$ session 4: intratumoral and peritumoral budding; ${ }^{2,20,21,47}$ session 5: field number and size for the tumor budding score;11,13,18,44-46,48,49 session 6: cut-offs and continuous scale for the tumor budding score; ${ }^{22}$ session 7: reporting tumor budding. ${ }^{11,12,18,44-46,49}$

\section{Grading of Recommendations Assessment, Development and Evaluation (GRADE)}

The quality of evidence and the strength of recommendations were based on the GRADE system: ${ }^{50-58}$ level of evidence:

High: further research is very unlikely to change our confidence in the estimate of effect.

Moderate: further research is likely to have an important impact on our confidence in the estimate of effect and may change the estimate.

Low: further research is very likely to have an important impact on our confidence in the estimate of effect and is likely to change the estimate.

Very low: any estimate of effect is very uncertain. Strength of recommendation:

Strong: for intervention: desirable effects outweigh undesirable effects; against intervention: undesirable effects outweigh desirable effects.

Weak: for intervention: desirable effects probably outweigh undesirable effects; against intervention: undesirable effects probably outweigh desirable effects.

\section{Results and Discussion}

\section{Recommendations}

Statement 1: tumor budding is defined as a single tumor cell or a cell cluster of up to 4 tumor cells. GRADE: strong recommendation, vote: $22 / 22$ $(100 \%)$, quality of evidence: high. Evidence and decision: most outcome data are based on studies defining tumor budding as single tumor cells or clusters of up to four tumor cells, although a cut-off of five cells has also been used.1,5 The prognostic power of tumor budding is not affected by the number of cells that defines a cluster. ${ }^{11,13-15,18}$ As four tumor cells is the most widely used cut-off for tumor budding, and as this cut-off distinguishes tumor budding from the novel histopathological parameter 'poorly differentiated cluster (PDC)', which is defined as five or more cells, ${ }^{27,59}$ the ITBCC 
group agreed on a cut-off of up to four cells to define tumor budding (Figure 1).

Statement 2: tumor budding is an independent predictor of lymph node metastasis in pT1 colorectal Cancer. GRADE: strong recommendation, vote: 23/23 (100\%), quality of evidence: high. Evidence and decision: in pT1 colorectal cancer, there is need for reliable predictors of local lymph node metastasis as their presence may help select candidates for radical surgery following endoscopic resection. Numerous studies, as well as four meta-analyses, have shown tumor budding to be a strong and independent predictor of local lymph node metastases in pT1 colorectal cancer. ${ }^{15-17,31-33,60-68}$ The ITBCC group therefore strongly recommends the reporting of tumor budding in pT1 colorectal cancer along with other histopathologic predictors of lymph node metastasis, such as poor differentiation, lymphovascular invasion and depth/level of submucosal invasion. ${ }^{16-18}$

Statement 3: tumor budding is an independent predictor of survival in stage II colorectal cancer. GRADE: strong recommendation, vote: 23/23 (100\%), quality of evidence: high. Evidence and decision: the UICC/AJCC TNM staging system remains the gold standard for prognostication in colorectal cancer and strongly influences adjuvant therapy decisions. Patients with stage III colorectal cancer are generally offered adjuvant chemotherapy, whereas those with stage II are not unless other highrisk features are present (ie, tumor perforation, lymphovascular invasion, serosal involvement (pT4a), poor tumor differentiation in microsatellite stable tumors, close/indeterminate/positive margins, perineural invasion and low lymph node yield).
However, some stage II colorectal cancer patients show worse survival than stage III colorectal cancer patients who receive adjuvant chemotherapy. ${ }^{69-71}$ Numerous studies and meta-analysis have shown tumor budding to be an independent predictor of recurrence and survival in stage II colorectal cancer, ${ }^{11,12,35,37-39,43,48,49}$ with outcomes similar to stage III colorectal cancer. ${ }^{39,72,73}$ Based on these data, the ITBCC group strongly recommends that tumor budding be included among the high-risk factors reported in stage II colorectal cancer.

Statement 4: tumor budding should be taken into account along with other clinicopathological factors in a multidisciplinary setting. GRADE: strong recommendation, vote: $23 / 23$ (100\%), quality of evidence: high. Evidence and decision: in addition to the TNM classification, prognostic factors such as tumor grade (G), histological subtype, vascular invasion $(\mathrm{V})$, perineural invasion (Pn) and margin status are routinely reported, in accordance with current guidelines and protocols. ${ }^{69-71}$ Molecular biomarkers include microsatellite, KRAS mutation and $B R A F$ mutation status. ${ }^{74}$ Based on available evidence, the ITBCC group considers the prognostic value of tumor budding to be at least equivalent to that of $\mathrm{V}, \mathrm{G}$ and Pn status, and therefore recommends that tumor budding (Bd) should be taken in to account along with these and other clinicopathologic factors in the risk assessment of colorectal cancer.

Statement 5: tumor budding is counted on H\&E. GRADE: strong recommendation, vote: 19/22 (86\%), quality of evidence: moderate. Evidence and decision: outcome data for tumor budding are largely based on studies using H\&E assessment. Relatively few studies have evaluated tumor budding by
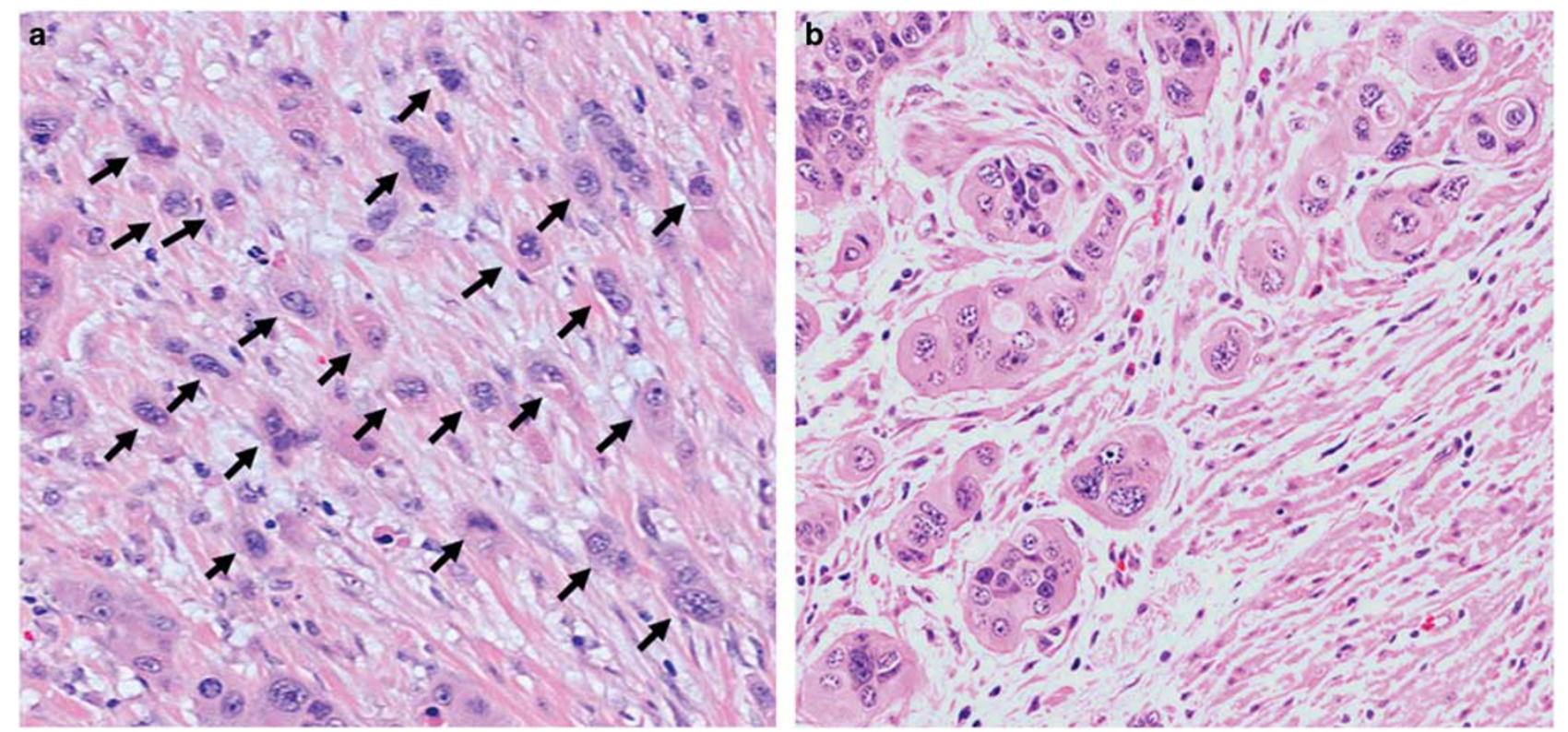

Figure 1 Example of tumor budding (a) that is defined as single tumor cells or tumor cell clusters at up to four cells. Example of poorly differentiated clusters (b) that are defined as five tumor cells or more. 
immunohistochemistry (IHC). ${ }^{13,14,18,45,75}$ Metaanalyses suggest that the prognostic power of tumor budding assessed on $\mathrm{H} \& \mathrm{E}$ and IHC do not differ materially. ${ }^{11,13-15,18}$ Some studies have found IHC to be superior to $\mathrm{H} \& \mathrm{E}$ with regard to reproducibility and inter-observer agreement, ${ }^{14,18,45}$ while others have not. ${ }^{41,48}$ The ITBCC group recommends that tumor budding should be evaluated on H\&E, as the vast majority outcome data are based on $\mathrm{H} \& \mathrm{E}$ assessment (particularly in pT1 colorectal cancer). In addition, the cost-effectiveness of $\mathrm{H} \& \mathrm{E}$ allows tumor budding to be assessed worldwide. This recommendation may change as more data on IHC assessment become available. It should be noted that tumor buds may be obscured by a peritumoral inflammatory infiltrate, making their identification difficult on H\&E. In addition, tumor buds may, on occasion, be difficult to distinguish from reactive stromal cells. In such scenarios, pankeratin IHC allows for better visualization of tumor buds, although it may also stain apoptotic bodies and cellular debris, which should not be counted as buds. Although the final tumor bud count is performed on $\mathrm{H} \& \mathrm{E}$, IHC can be helpful in challenging cases (ie, glandular fragmentation, strong peritumoral inflammation) to confirm that the cells being counted are indeed tumor buds.

Statement 6: ITB in colorectal cancer has been shown to be related to lymph node metastasis. GRADE: strong recommendation, vote: $22 / 22$ (100\%), quality of evidence: low. Evidence and decision: in contrast to conventional or so-called 'peritumoral budding' (PTB), which is seen at the invasive front, ITB refers to budding within the main tumor body. Although the terms ITB and PTB were introduced in 2011, ${ }^{2}$ ITB was first reported in 1989 in a series in rectal cancer biopsies and found to be associated with lymph node metastases. ${ }^{23}$ More recently, ITB in pre-operative biopsies has been shown to correlate with high-grade PTB, lymph node

\begin{tabular}{|c|c|c|}
\hline \multicolumn{2}{|c|}{ Objective magnification: 20} \\
\hline $\begin{array}{c}\text { Eyepiece FN } \\
\text { Diameter }(\mathrm{mm})\end{array}$ & $\begin{array}{c}\text { Specimen Area } \\
(\mathrm{mm} 2)\end{array}$ & $\begin{array}{c}\text { Normalization } \\
\text { Factor }\end{array}$ \\
\hline 18 & 0.636 & 0.810 \\
\hline 19 & 0.709 & 0.903 \\
\hline 20 & 0.785 & 1.000 \\
\hline 21 & 0.866 & 1.103 \\
\hline 22 & 0.950 & 1.210 \\
\hline 23 & 1.039 & 1.323 \\
\hline 24 & 1.131 & 1.440 \\
\hline 25 & 1.227 & 1.563 \\
\hline 26 & 1.327 & 1.690 \\
\hline
\end{tabular}

Figure 2 Conversion table to adjust and standardize the tumor bud count for different microscope types. metastases and tumor regression grade in the corresponding colorectal cancer resection specimens. ${ }^{20-22}$ Although ITB may prove to be a promising biomarker in the pre-operative management of colorectal cancer patients, there is insufficient evidence to support its routine reporting at this time. The ITBCC group therefore recommends further research in this area before reporting of ITB is implemented in routine practice.

Statement 7: tumor budding is assessed in one hotspot (in a field measuring $0.785 \mathrm{~mm}^{2}$ ) at the invasive front. GRADE: strong recommendation, vote: $22 / 22$ (100\%), quality of evidence: moderate. Evidence and decision: standardization is crucial for the implementation of any biomarker in clinical practice. Several different scoring systems for tumor budding are currently in use as highlighted by recent reviews, meta-analyses and the ITBCC pre-meeting survey. ${ }^{11-16,18}$ Although the prognostic significance of tumor budding is largely independent of the scoring system used, the presence of a single international standard is critical for future clinical trials and diagnostic practice. To ensure standardization of field size, the ITBCC group recommends reporting by area (ie, $\mathrm{mm}^{2}$ ) rather than objective lens (eg, 20x), as the field of vision varies widely between different microscopes. The field area selected by the ITBCC group is $0.785 \mathrm{~mm}^{2}$, which corresponds to the field area used by Ueno et $a l^{17,63}$ and adopted by the Japanese Society for Cancer of the Colon and Rectum $^{76}$ (20x objective lens with a $20 \mathrm{~mm}$ eyepiece field number diameter). A conversion table has been developed to normalize bud counts to $0.785 \mathrm{~mm}^{2}$ for microscopes with ocular lenses associated with different fields of vision (Figure 2).

Statement 8: for tumor budding assessment in colorectal cancer, the hotspot method is recommended. GRADE: strong recommendation, vote: $22 / 22(100 \%)$, quality of evidence: moderate. Evidence and decision: most studies have performed tumor bud counts in a single field with the highest density of tumor buds ('hotspot' method), whereas others have used multiple fields (eg, ' 5 high power field' and '10 high power field' methods). 11-16,18 Counting across multiple fields has the advantage of being more representative of the entire invasive front, and there is also some evidence of improved inter-observer agreement using this approach. ${ }^{11-16,18,45}$ On the other hand, counting multiple fields may 'dilute' the final (mean) tumor bud count in cases with focally many tumor buds. The 'hotspot' method therefore better reflects the maximal extent of tumor budding at the invasive front. The ITBCC group recommends the use of the 'hotspot' method, as this is the method used in the vast majority of outcome based studies, and interobserver agreement using this method is quite acceptable. ${ }^{11-16,18,41,48}$ However, to ensure that the field with the highest tumor budding is selected, it is 

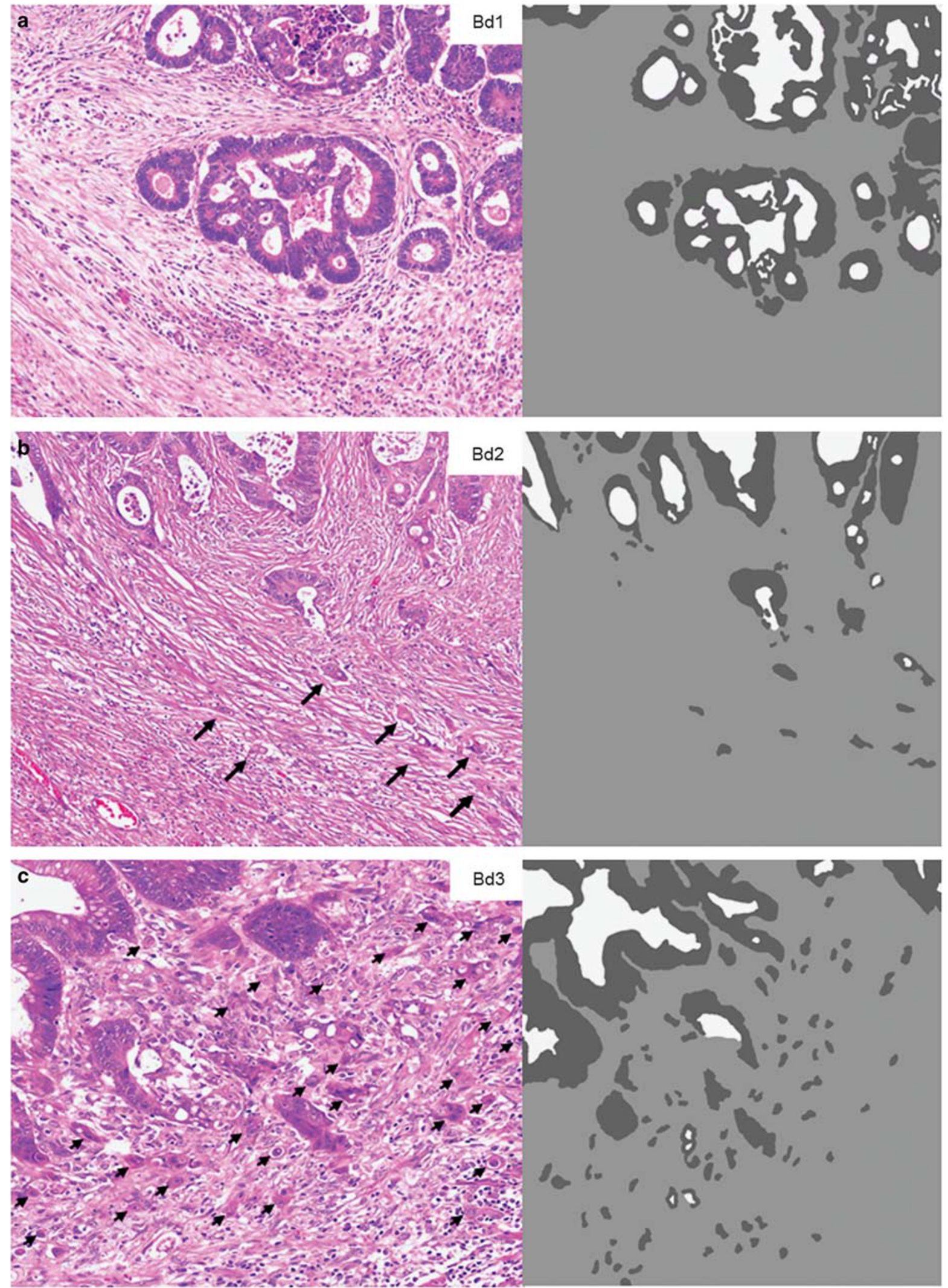

Figure 3 Examples of different tumor budding grades (hotspot, $0.785 \mathrm{~mm}^{2}$ ) at the invasive front of colorectal cancer based on the ITBCC 2016. (a): Bd 1 (low), (b): Bd 2 (intermediate) and (c): Bd 3 (high). 
1 Define the field (specimen) area for the $20 x$ objective lens of your microscope based on the eyepiece field number (FN) diameter

\begin{tabular}{|c|c|c|}
\hline \multicolumn{2}{|c|}{ Objective magnification: 20} \\
\hline $\begin{array}{c}\text { Eyepiece FN } \\
\text { Diameter (mm) }\end{array}$ & $\begin{array}{c}\text { Specimen Area } \\
(\mathrm{mm} 2)\end{array}$ & $\begin{array}{c}\text { Normalization } \\
\text { Factor }\end{array}$ \\
\hline 18 & 0.636 & 0.810 \\
\hline 19 & 0.709 & 0.903 \\
\hline 20 & 0.785 & 1.000 \\
\hline 21 & 0.866 & 1.103 \\
\hline 22 & 0.950 & 1.210 \\
\hline 23 & 1.039 & 1.323 \\
\hline 24 & 1.131 & 1.440 \\
\hline 25 & 1.227 & 1.563 \\
\hline 26 & 1.327 & 1.690 \\
\hline
\end{tabular}

2 Select the H\&E slide with greatest degree of budding at the invasive front

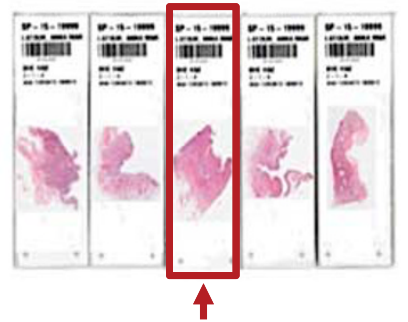

3 Scan 10 individual fields at medium power (10x objective) to identify the "hotspot" at the invasive front

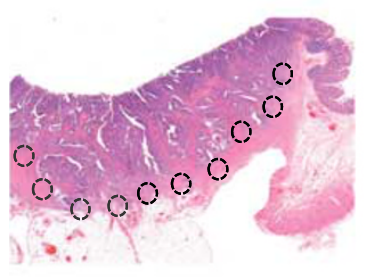

For surgical resection specimens, scan 10 fields

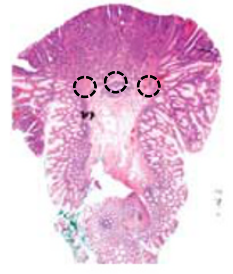

For pT1 endoscopic resections (usually $<10$ fields available), scan all

4

Count tumor buds in the selected "hotspot" (20x objective)

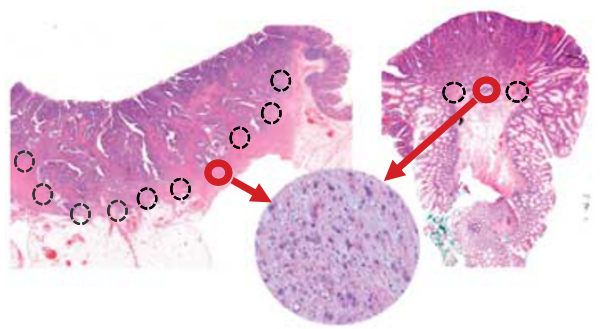

Selected hotspot indicated in red

Divide the bud count by the normalization factor (figure 2) to determine the tumor bud count per $0.785 \mathrm{~mm}^{2}$

Select the budding [Bd] category based on bud count and indicate the absolute count per $0.785 \mathrm{~mm}^{2}$ (see reporting example)

\begin{tabular}{|c|c|c|}
\hline \multirow{2}{*}{$\begin{array}{l}\text { Tumor bud count } \\
\text { per } 0.785 \mathrm{~mm}^{2}=\end{array}$} & \multicolumn{2}{|c|}{ Bud count (20x objective) } \\
\hline & Norma & zation factor* \\
\hline $\begin{array}{l}\text { Bd1 (low): } \\
\text { Bd2 (intermediate): } \\
\text { Bd3 (high): }\end{array}$ & $\begin{array}{l}0-4 \text { buds } \\
5-9 \text { buds } \\
\geq 10 \text { buds }\end{array}$ & per $0.785 \mathrm{~mm}^{2}$ \\
\hline
\end{tabular}

Reporting example:

Tumor budding: Bd3 (high), count 14 (per $0.785 \mathrm{~mm}^{2}$ )

Figure 4 Procedure proposed by the ITBCC 2016 for reporting tumor budding in colorectal cancer in daily diagnostic practice. 
Table 2 Statements of the ITBCC 2016 based on the GRADE system

\begin{tabular}{|c|c|c|c|}
\hline & \multirow{2}{*}{ Statement } & \multicolumn{2}{|l|}{ Grade } \\
\hline & & Recommendation & Evidence \\
\hline 1 & $\begin{array}{l}\text { Tumor budding is defined } \\
\text { as a single tumor cell or a } \\
\text { cell cluster consisting of } \\
\text { four tumor cells or less }\end{array}$ & $\begin{array}{l}\text { Strong } \\
\text { Vote: } 22 / 22(100 \%)\end{array}$ & High \\
\hline 2 & $\begin{array}{l}\text { Tumor budding is an } \\
\text { independent predictor of } \\
\text { lymph node metastasis in } \\
\text { pT1 colorectal cancer }\end{array}$ & $\begin{array}{l}\text { Strong } \\
\text { Vote: } 23 / 23(100 \%)\end{array}$ & High \\
\hline 3 & $\begin{array}{l}\text { Tumor budding is an } \\
\text { independent predictor of } \\
\text { survival in stage II } \\
\text { colorectal cancer }\end{array}$ & $\begin{array}{l}\text { Strong } \\
\text { Vote: } 23 / 23(100 \%)\end{array}$ & High \\
\hline 4 & $\begin{array}{l}\text { Tumor budding should be } \\
\text { taken into account along } \\
\text { with other } \\
\text { clinicopathological features } \\
\text { in a multidisciplinary } \\
\text { setting }\end{array}$ & $\begin{array}{l}\text { Strong } \\
\text { Vote: } 23 / 23(100 \%)\end{array}$ & High \\
\hline 5 & $\begin{array}{l}\text { Tumor budding is counted } \\
\text { on H\&E }\end{array}$ & $\begin{array}{l}\text { Strong } \\
\text { Vote: } 19 / 22(86 \%)\end{array}$ & Moderate \\
\hline 6 & $\begin{array}{l}\text { Intratumoral budding exists } \\
\text { in colorectal cancer and has } \\
\text { been shown to be related to } \\
\text { lymph node metastasis }\end{array}$ & $\begin{array}{l}\text { Strong } \\
\text { Vote: } 22 / 22(100 \%)\end{array}$ & Low \\
\hline 7 & $\begin{array}{l}\text { Tumor budding is assessed } \\
\text { in one hotspot (in a field } \\
\text { measuring } 0.785 \mathrm{~mm}^{2} \text { ) at } \\
\text { the invasive front }\end{array}$ & $\begin{array}{l}\text { Strong } \\
\text { Vote: } 22 / 22(100 \%)\end{array}$ & Moderate \\
\hline 8 & $\begin{array}{l}\text { For tumor budding } \\
\text { assessment in colorectal } \\
\text { cancer, the hotspot method } \\
\text { is recommended }\end{array}$ & $\begin{array}{l}\text { Strong } \\
\text { Vote: } 22 / 22(100 \%)\end{array}$ & Moderate \\
\hline 9 & $\begin{array}{l}\text { A three-tier system should } \\
\text { be used along with the } \\
\text { budding count in order to } \\
\text { facilitate risk stratification } \\
\text { in colorectal cancer }\end{array}$ & $\begin{array}{l}\text { Strong } \\
\text { Vote: } 23 / 23(100 \%)\end{array}$ & Moderate \\
\hline 10 & $\begin{array}{l}\text { Tumor budding should be } \\
\text { included in guidelines/ } \\
\text { protocols for colorectal } \\
\text { cancer reporting }\end{array}$ & $\begin{array}{l}\text { Strong } \\
\text { Vote: } 23 / 23(100 \%)\end{array}$ & High \\
\hline 11 & $\begin{array}{l}\text { Tumor budding and tumor } \\
\text { grade are not the same }\end{array}$ & $\begin{array}{l}\text { Strong } \\
\text { Vote: } 23 / 23(100 \%)\end{array}$ & High \\
\hline
\end{tabular}

recommended that 10 separate fields (20x objective) along the invasive front are scanned before counting of tumor buds in the single selected 'hotspot'.

Statement 9: a three-tier system should be used along with the budding count in order to facilitate risk stratification in colorectal cancer. GRADE: strong recommendation, vote: $23 / 23$ (100\%), quality of evidence: moderate. Evidence and decision: for risk stratification based on tumor bud counts, most studies have used numerical cut-offs (including twotier and three-tier systems), while a few studies have used a continuous scale to predict probability of recurrence. ${ }^{11-16,18}$ As tumor budding behaves as a continuous variable, a continuous scale provides more precise risk stratification than does a numerical cut-off. ${ }^{22}$ Nonetheless, cut-offs are more practical in the clinical setting and there is insufficient evidence to support the use of a continuous scale for tumor budding in clinical decision making. The ITBCC group recommends the use of a three-tier system as used by Japanese Society for Cancer of the Colon and Rectum: ${ }^{77}$

- 0-4 buds-low budding (Bd 1).

- 5-9 buds-intermediate budding ( $\mathrm{Bd} \mathrm{2).}$

- 10 or more buds—high budding (Bd 3).

This system allows for risk stratification of both pT1 colorectal cancer and stage II colorectal cancer. In pT1 colorectal cancer, $\mathrm{Bd} 2$ and $\mathrm{Bd} 3$ are associated with an increased risk of lymph node metastasis $^{32,61,63,65,68,78-81}$ whereas in stage II colorectal cancer, Bd 3 is associated with an increased risk of recurrence and mortality. ${ }^{15,16,31,37,67,82-85}$ The ITBCC group recommends that, in addition to the $\mathrm{Bd}$ category, the absolute bud count is provided (eg, $\mathrm{Bd}$ 3 (count 17)). This avoids loss of information that may occur when applying a cut-off to borderline cases. For example, a bud count of 9 (Bd 2) may be biologically similar to a bud count of 10 (Bd 3), but falls into a different risk category. As indicated in statement 4 , it is important that tumor budding is taken into account along with other clinicopathological features in a multidisciplinary setting. Histological examples of budding 1, 2 and 3 are represented in Figure 3.

Statement 10: tumor budding should be included in guidelines/protocols for colorectal cancer reporting GRADE: strong recommendation, vote: $23 / 23$ (100\%), quality of evidence: high. Evidence and decision: tumor budding is a well-established, independent prognostic factor in colorectal cancer with the potential to impact clinical decision making in pT1 and stage II disease (see statements 2 and 3). ${ }^{11-16,18}$ The standardized, international, evidence-based method for tumor budding assessment agreed on at the ITBCC (Figure 4), provides a basis for future reporting of tumor budding in routine practice. The ITBCC group therefore recommends that tumor budding should be included in guidelines and protocols for the pathology reporting of colorectal cancer.

Statement 11: tumor budding and tumor grade are not the same GRADE: Strong recommendation, vote: $23 / 23(100 \%)$, quality of evidence: high. Evidence and decision: tumor budding is defined by the presence of single cells or clusters of up to four cells at the invasive front (see statement 1), whereas tumor grade is defined by the proportion of tumor demonstrating gland formation. In multivariate analyses, the prognostic effect of tumor budding is independent of tumor grade and growth pattern. ${ }^{1,11-16,18}$ Therefore, the ITBCC group considers tumor 
budding to be different from tumor grade, and to have prognostic value that is independent of tumor grade.

All the statements and the corresponding GRADE recommendation and evidence are summarized in Table 2 .

\section{Additional Practical Aspects for Tumor Budding in Colorectal Cancer}

In some histological subtypes of colorectal cancer (eg, mucinous, signet-ring cell, medullary and micropapillary), the assessment of tumor budding should be performed with caution. In mucinous and signet-ring cell carcinomas, tumor buds suspended in pools of mucin should not be counted. In medullary carcinomas, discohesion or separation of tumor cells secondary to inflammation may be impossible to distinguish from true tumor buds. In micropapillary carcinoma, care should be taken not to include poorly differentiated clusters in the tumor bud count (see statement 1). Finally, glandular fragmentation secondary to heavy (often neutrophil rich) inflammation may be difficult to distinguish from tumor budding. In cases where an accurate tumor bud count cannot be performed, Bd can be reported as 'cannot be assessed' with an explanatory note. In rectal cancer resections after neo-adjuvant therapy, tumor budding should not be reported as there are insufficient data regarding its prognostic significance. ${ }^{86-89}$

\section{Conclusion and future perspectives}

Tumor budding is a well-established prognostic factor with the potential to refine clinical management decisions in patients with colorectal cancer. Consensus on a standardized, evidence-based method for tumor budding assessment at the ITBCC paves the way for future reporting of tumor budding in routine practice. The ITBCC is not intended to be an end-point, but rather a foundation for further multi-center collaborations and clinical trials to prospectively validate and further refine the proposed ITBCC method. The ITBCC recommends that tumor budding should be included in future colorectal cancer guidelines and protocols.

\section{Acknowledgments}

The ITBCC participants thank all the sponsors for logistic support (in alphabetic order: AstraZeneca, Basys Data, Econis, Formafix, Medite, Nikon, Sakura, SPOT Imaging, 3DHisttech, Visiopharm), Philip Merlo, SPOT Imaging, for providing the conversion table and Tilman Schulz, MD (Institute of Pathology, Klinikum Bayreuth, Germany) for providing us with the schemes in Figure 3. PQ is supported by program grants from Yorkshire Cancer Research.

\section{Disclosure/conflict of interest}

The authors declare no conflict of interest.

\section{References}

1 Ueno H, Murphy J, Jass JR, et al. Tumour 'budding' as an index to estimate the potential of aggressiveness in rectal cancer. Histopathology 2002;40:127-132.

2 Lugli A, Vlajnic T, Giger O, et al. Intratumoral budding as a potential parameter of tumor progression in mismatch repair-proficient and mismatch repairdeficient colorectal cancer patients. Hum Pathol 2011;42:1833-1840.

3 De Smedt L, Palmans S, Andel D, et al. Expression profiling of budding cells in colorectal cancer reveals an EMT-like phenotype and molecular subtype switching. Br J Cancer 2016;116:58-65.

4 Jass JR, Barker M, Fraser L, et al. APC mutation and tumour budding in colorectal cancer. J Clin Pathol 2003;56:69-73.

5 Zlobec I, Lugli A, Baker K, et al. Role of APAF-1, E-cadherin and peritumoral lymphocytic infiltration in tumour budding in colorectal cancer. J Pathol 2007;212: 260-268.

6 Karamitopoulou E, Lugli A, Panayiotides I, et al. Systematic assessment of protein phenotypes characterizing high-grade tumour budding in mismatch repair-proficient colorectal cancer. Histopathology 2010;57:233-243.

7 Dawson H, Koelzer VH, Karamitopoulou E, et al. The apoptotic and proliferation rate of tumour budding cells in colorectal cancer outlines a heterogeneous population of cells with various impacts on clinical outcome. Histopathology 2014;64:577-584.

8 Prall F, Ostwald C, Weirich V, et al. p16(INK4a) promoter methylation and 9p21 allelic loss in colorectal carcinomas: relation with immunohistochemical p16(INK4a) expression and with tumor budding. Hum Pathol 2006;37:578-585.

9 Zlobec I, Lugli A. Epithelial mesenchymal transition and tumor budding in aggressive colorectal cancer: tumor budding as oncotarget. Oncotarget 2010;1:651-661.

10 Hostettler L, Zlobec I, Terracciano L, et al. ABCG5positivity in tumor buds is an indicator of poor prognosis in node-negative colorectal cancer patients. World J Gastroenterol 2010;16:732-739.

11 Mitrovic B, Schaeffer DF, Riddell RH, et al. Tumor budding in colorectal carcinoma: time to take notice. Mod Pathol 2012;25:1315-1325.

12 Lugli A, Karamitopoulou E, Zlobec I. Tumour budding: a promising parameter in colorectal cancer. Br J Cancer 2012;106:1713-1717.

13 van Wyk HC, Park J, Roxburgh C, et al. The role of tumour budding in predicting survival in patients with primary operable colorectal cancer: a systematic review. Cancer Treat Rev 2015;41:151-159.

14 De Smedt L, Palmans S, Sagaert X. Tumour budding in colorectal cancer: what do we know and what can we do? Virchows Arch 2016;468:397-408.

15 Rogers AC, Winter DC, Heeney A, et al. Systematic review and meta-analysis of the impact of tumour budding in colorectal cancer. Br J Cancer 2016;115:831-840.

16 Bosch SL, Teerenstra S, de Wilt $\mathrm{H}$, et al. Predicting lymph node metastasis in pT1 colorectal cancer: 
a systematic review of risk factors providing rationale for therapy decisions. Endoscopy 2013;45:827-834.

17 Ueno H, Mochizuki H, Hashiguchi Y, et al. Risk factors for an adverse outcome in early invasive colorectal carcinoma. Gastroenterology 2004;127:385-394.

18 Koelzer VH, Zlobec I, Lugli A. Tumor budding in colorectal cancer-ready for diagnostic practice? Hum Pathol 2016;47:4-19.

19 Wang LM, Kevans D, Mulcahy H, et al. Tumor budding is a strong and reproducible prognostic marker in T3N0 colorectal cancer. Am J Surg Pathol 2009;33:134-141.

20 Giger OT, Comtesse SC, Lugli A, et al. Intra-tumoral budding in preoperative biopsy specimens predicts lymph node and distant metastasis in patients with colorectal cancer. Mod Pathol 2012;25:1048-1053.

21 Rogers AC, Gibbons D, Hanly AM, et al. Prognostic significance of tumor budding in rectal cancer biopsies before neoadjuvant therapy. Mod Pathol 2014;27:156-162.

22 Zlobec I, Hadrich M, Dawson $\mathrm{H}$, et al. Intratumoural budding (ITB) in preoperative biopsies predicts the presence of lymph node and distant metastases in colon and rectal cancer patients. Br J Cancer 2014;110: 1008-1013.

23 Morodomi T, Isomoto $\mathrm{H}$, Shirouzu K, et al. An index for estimating the probability of lymph node metastasis in rectal cancers. Lymph node metastasis and the histopathology of actively invasive regions of cancer. Cancer 1989;63:539-543.

24 Hase K, Shatney C, Johnson D, et al. Prognostic value of tumor 'budding' in patients with colorectal cancer. Dis Colon Rectum 1993;36:627-635.

25 Shinto E, Mochizuki H, Ueno H, et al. A novel classification of tumour budding in colorectal cancer based on the presence of cytoplasmic pseudo-fragments around budding foci. Histopathology 2005;47:25-31.

26 Okuyama T, Oya M, Yamaguchi M. Budding (sprouting) as a useful prognostic marker in colorectal mucinous carcinoma. Jpn J Clin Oncol 2002;32:412-416.

27 Ueno H, Kajiwara Y, Shimazaki H, et al. New criteria for histologic grading of colorectal cancer. Am J Surg Pathol 2012;36:193-201.

28 Greco P, Magro G. Comments on the reporting of lymph nodes and tumour budding in the checklist of colorectal carcinoma. Virchows Arch 2007;450: 359-360.

29 Pusiol T, Piscioli F, Zorzi MG, et al. Accurate and reproducible definition of tumor budding as prognostic marker of colon cancer. Dis Colon Rectum 2010;53: 1576 author reply.

30 Prall F. Tumour budding in colorectal carcinoma. Histopathology 2007;50:151-162.

31 Beaton C, Twine CP, Williams GL, et al. Systematic review and meta-analysis of histopathological factors influencing the risk of lymph node metastasis in early colorectal cancer. Colorectal Dis 2013;15:788-797.

32 Kawachi $\mathrm{H}$, Eishi $\mathrm{Y}$, Ueno $\mathrm{H}$, et al. A three-tier classification system based on the depth of submucosal invasion and budding/sprouting can improve the treatment strategy for T1 colorectal cancer: a retrospective multicenter study. Mod Pathol 2015;28:872-879.

33 Kajiwara Y, Ueno H, Hashiguchi Y, et al. Risk factors of nodal involvement in T2 colorectal cancer. Dis Colon Rectum 2010;53:1393-1399.

34 Wang HS, Liang WY, Lin TC, et al. Curative resection of T1 colorectal carcinoma: risk of lymph node metastasis and long-term prognosis. Dis Colon Rectum 2005;48: 1182-1192.
35 Betge J, Kornprat P, Pollheimer MJ, et al. Tumor budding is an independent predictor of outcome in AJCC/UICC stage II colorectal cancer. Ann Surg Oncol 2012;19:3706-3712.

36 Canney AL, Kevans D, Wang LM, et al. Stage II colonic adenocarcinoma: a detailed study of pT4No with emphasis on peritoneal involvement and the role of tumour budding. Histopathology 2012;61:488-496.

37 Petrelli F, Pezzica E, Cabiddu M, et al. Tumour budding and survival in stage II colorectal cancer: a systematic review and pooled analysis. J Gastrointest Cancer 2015;46:212-218.

38 Lai YH, Wu LC, Li PS, et al. Tumour budding is a reproducible index for risk stratification of patients with stage II colon cancer. Colorectal Dis 2014;16:259-264.

39 Nakamura T, Mitomi H, Kanazawa H, et al. Tumor budding as an index to identify high-risk patients with stage II colon cancer. Dis Colon Rectum 2008;51: $568-572$.

40 Prall F, Nizze H, Barten M. Tumour budding as prognostic factor in stage I/II colorectal carcinoma. Histopathology 2005;47:17-24.

41 Kai K, Aishima S, Aoki S, et al. Cytokeratin immunohistochemistry improves interobserver variability between unskilled pathologists in the evaluation of tumor budding in T1 colorectal cancer. Pathol Int 2016;66:75-82.

42 Ohtsuki K, Koyama F, Tamura T, et al. Prognostic value of immunohistochemical analysis of tumor budding in colorectal carcinoma. Anticancer Res 2008;28: 1831-1836.

43 Hayes BD, Maguire A, Conlon N, et al. Reproducibility of the rapid bud count method for assessment of tumor budding in stage II colorectal cancer. Am J Surg Pathol 2010;34:746-748.

44 Karamitopoulou E, Zlobec I, Kolzer V, et al. Proposal for a 10-high-power-fields scoring method for the assessment of tumor budding in colorectal cancer. Mod Pathol 2013;26:295-301.

45 Koelzer VH, Zlobec I, Berger MD, et al. Tumor budding in colorectal cancer revisited: results of a multicenter interobserver study. Virchows Arch 2015;466:485-493.

46 Puppa G, Senore C, Sheahan K, et al. Diagnostic reproducibility of tumour budding in colorectal cancer: a multicentre, multinational study using virtual microscopy. Histopathology 2012;61:562-575.

47 Zlobec I, Borner M, Lugli A, et al. Role of intra- and peritumoral budding in the interdisciplinary management of rectal cancer patients. Int J Surg Oncol 2012;2012:795945.

48 Graham RP, Vierkant RA, Tillmans LS, et al. Tumor budding in colorectal carcinoma: confirmation of prognostic significance and histologic cutoff in a populationbased cohort. Am J Surg Pathol 2015;39:1340-1346.

49 Horcic M, Koelzer VH, Karamitopoulou E, et al. Tumor budding score based on 10 high-power fields is a promising basis for a standardized prognostic scoring system in stage II colorectal cancer. Hum Pathol 2013;44:697-705.

50 Guyatt G, Oxman AD, Akl EA, et al. GRADE guidelines: 1. Introduction-GRADE evidence profiles and summary of findings tables. J Clin Epidemiol 2011;64:383-394.

51 Guyatt GH, Oxman AD, Kunz R, et al. GRADE guidelines: 2. Framing the question and deciding on important outcomes. J Clin Epidemiol 2011;64:395-400.

52 Guyatt GH, Oxman AD, Kunz R, et al. GRADE guidelines 6. Rating the quality of evidence-imprecision. J Clin Epidemiol 2011;64:1283-1293. 
53 Guyatt GH, Oxman AD, Kunz R, et al. GRADE guidelines: 8. Rating the quality of evidence-indirectness. J Clin Epidemiol 2011;64:1303-1310.

54 Guyatt GH, Oxman AD, Kunz R, et al. GRADE guidelines: 7. Rating the quality of evidence-inconsistency. J Clin Epidemiol 2011;64:1294-1302.

55 Guyatt GH, Oxman AD, Montori V, et al. GRADE guidelines: 5. Rating the quality of evidencepublication bias. J Clin Epidemiol 2011;64:1277-1282.

56 Guyatt GH, Oxman AD, Schunemann HJ, et al. GRADE guidelines: a new series of articles in the Journal of Clinical Epidemiology. J Clin Epidemiol 2011;64:380-382.

57 Guyatt GH, Oxman AD, Sultan S, et al. GRADE guidelines: 9. Rating up the quality of evidence. J Clin Epidemiol 2011;64:1311-1316.

58 Guyatt GH, Oxman AD, Vist G, et al. GRADE guidelines: 4. Rating the quality of evidence-study limitations (risk of bias). J Clin Epidemiol 2011;64:407-415.

59 Barresi V, Branca G, Ieni A, et al. Poorly differentiated clusters (PDCs) as a novel histological predictor of nodal metastases in pT1 colorectal cancer. Virchows Arch 2014;464:655-662.

60 Kitajima K, Fujimori T, Fujii S, et al. Correlations between lymph node metastasis and depth of submucosal invasion in submucosal invasive colorectal carcinoma: a Japanese collaborative study. J Gastroenterol 2004;39:534-543.

61 Kaneko I, Tanaka S, Oka S, et al. Immunohistochemical molecular markers as predictors of curability of endoscopically resected submucosal colorectal cancer. World J Gastroenterol 2007;13:3829-3835.

62 Tateishi Y, Nakanishi Y, Taniguchi H, et al. Pathological prognostic factors predicting lymph node metastasis in submucosal invasive (T1) colorectal carcinoma. Mod Pathol 2010;23:1068-1072.

63 Ueno H, Hashiguchi Y, Kajiwara Y, et al. Proposed objective criteria for 'grade 3' in early invasive colorectal cancer. Am J Clin Pathol 2010;134:312-322.

64 Keum MA, Lim SB, Kim SA, et al. Clinicopathologic factors affecting recurrence after curative surgery for stage I colorectal cancer. J Korean Soc Coloproctol 2012;28:49-55.

65 Nakadoi K, Tanaka S, Kanao H, et al. Management of T1 colorectal carcinoma with special reference to criteria for curative endoscopic resection. J Gastroenterol Hepatol 2012;27:1057-1062.

66 Suh JH, Han KS, Kim BC, et al. Predictors for lymph node metastasis in $\mathrm{T} 1$ colorectal cancer. Endoscopy 2012;44:590-595.

67 Wada H, Shiozawa M, Katayama K, et al. Systematic review and meta-analysis of histopathological predictive factors for lymph node metastasis in $\mathrm{T} 1$ colorectal cancer. J Gastroenterol 2015;50:727-734.

68 Ueno H, Hase K, Hashiguchi Y, et al. Novel risk factors for lymph node metastasis in early invasive colorectal cancer: a multi-institution pathology review. J Gastroenterol 2014;49:1314-1323.

69 Quirke P, West NP, Nagtegaal ID. EURECCA consensus conference highlights about colorectal cancer clinical management: the pathologists expert review. Virchows Arch 2014;464:129-134.

70 van de Velde CJ, Aristei C, Boelens PG, et al. EURECCA colorectal: multidisciplinary mission statement on better care for patients with colon and rectal cancer in Europe. Eur J Cancer 2013;49:2784-2790.

71 van de Velde CJ, Boelens PG, Borras JM, et al. EURECCA colorectal: multidisciplinary management:
European consensus conference colon \& rectum. Eur J Cancer 2014;50:1 e-e34.

72 Okuyama T, Nakamura T, Yamaguchi M. Budding is useful to select high-risk patients in stage II welldifferentiated or moderately differentiated colon adenocarcinoma. Dis Colon Rectum 2003;46: 1400-1406.

73 Okuyama T, Oya M, Ishikawa H. Budding as a useful prognostic marker in pT3 well- or moderatelydifferentiated rectal adenocarcinoma. J Surg Oncol 2003;83:42-47.

74 Pritchard CC, Grady WM. Colorectal cancer molecular biology moves into clinical practice. Gut 2011;60:116-129.

75 Max N, Harbaum L, Pollheimer MJ, et al. Tumour budding with and without admixed inflammation: two different sides of the same coin? Br J Cancer 2016;114: $368-371$.

76 Watanabe T, Itabashi M, Shimada Y, et al. Japanese society for cancer of the colon and rectum (JSCCR) guidelines 2014 for treatment of colorectal cancer. Int J Clin Oncol 2015;20:207-239.

77 Tomita S, Yamauchi M, Ichikawa K, et al. [The brand new trend of colorectal carcinoma pathology]. Nihon Rinsho 2014;72:63-70.

78 Miyachi H, Kudo SE, Ichimasa K, et al. Management of T1 colorectal cancers after endoscopic treatment based on the risk stratification of lymph node metastasis. J Gastroenterol Hepatol 2016;31:1126-1132.

79 Nishida T, Egashira Y, Akutagawa H, et al. Predictors of lymph node metastasis in T1 colorectal carcinoma: an immunophenotypic analysis of 265 patients. Dis Colon Rectum 2014;57:905-915.

80 Okamura T, Shimada Y, Nogami H, et al. Tumor budding detection by immunohistochemical staining is not superior to hematoxylin and eosin staining for predicting lymph node metastasis in pT1 colorectal cancer. Dis Colon Rectum 2016;59:396-402.

81 Pai RK, Chen Y, Jakubowski MA, et al. Colorectal carcinomas with submucosal invasion (pT1): analysis of histopathological and molecular factors predicting lymph node metastasis. Mod Pathol 2017;30:113-122.

82 Choi JY, Jung SA, Shim KN, et al. Meta-analysis of predictive clinicopathologic factors for lymph node metastasis in patients with early colorectal carcinoma. J Korean Med Sci 2015;30:398-406.

83 Di Gregorio C, Bonetti LR, de Gaetani C, et al. Clinical outcome of low- and high-risk malignant colorectal polyps: results of a population-based study and metaanalysis of the available literature. Intern Emerg Med 2014;9:151-160.

84 Glasgow SC, Bleier JI, Burgart LJ, et al. Meta-analysis of histopathological features of primary colorectal cancers that predict lymph node metastases. J Gastrointest Surg 2012;16:1019-1028.

85 Mou S, Soetikno R, Shimoda T, et al. Pathologic predictive factors for lymph node metastasis in submucosal invasive (T1) colorectal cancer: a systematic review and meta-analysis. Surg Endosc 2013;27:2692-2703.

86 Kinoshita H, Watanabe T, Yanagisawa A, et al. Pathological changes of advanced lower-rectal cancer by preoperative radiotherapy. Hepatogastroenterology 2004;51:1362-1366.

87 Huebner M, Wolff BG, Smyrk TC, et al. Partial pathologic response and nodal status as most significant prognostic factors for advanced rectal cancer treated with preoperative chemoradiotherapy. World J Surg 2012;36:675-683. 
88 Du C, Xue W, Li J, et al. Morphology and prognostic value of tumor budding in rectal cancer after neoadjuvant radiotherapy. Hum Pathol 2012;43: 1061-1067.
89 Sannier A, Lefevre JH, Panis Y, et al. Pathological prognostic factors in locally advanced rectal carcinoma after neoadjuvant radiochemotherapy: analysis of 113 cases. Histopathology 2014;65:623-630. 